\title{
Intraoperative Application of Resuscitative endovascular balloon occlusion of the aorta (REBOA) to Traumatic Vascular Injury
}

\author{
Ji Wool Ko, Pil Young Jung \\ Department of Surgery, Yonsei University Wonju College of Medicine, Wonju Severance Christian Hospital, Regional trauma center
}

Resuscitative endovascular balloon occlusion of the aorta (REBOA) is effective in controlling massive bleeding caused by trauma (1). For this reason, REBOA is performed mainly in prehospital areas or emergency rooms until final hemostasis is performed (2). It requires great skill and can cause various complications (1). Nevertheless, the procedure may be optimal for achieving desirable outcomes. (3) In some studies, REBOA has been performed during surgery (4). We report a case in which REBOA was performed intraoperatively, and I describe the results.

(Trauma Image Proced 2019(1):12-14)

Key Words: Intraoperative, Resuscitative endovascular balloon occlusion of the aorta (REBOA), Balloon, Traumatic

\section{CASE}

A 63-year-old woman was in a car accident; she had been sitting in the passenger seat. In the emergency room, the patient's blood pressure was 82/46 $\mathrm{mm} \mathrm{Hg}$, the pulse rate was 84 beats/min, and her level of consciousness was normal. The lower abdomen was bruised with a seat belt sign (Fig. 1.). The extended focused assessment sonography in trauma showed a moderate to severe intra-abdominal fluid collection in Morison's pouch and in perivesical sites. The hemoglobin level was $8.8 \mathrm{~g} / \mathrm{dL}$, and the lactate level was $1.82 \mathrm{mmol} / \mathrm{L}$. No further imaging tests were performed, and exploratory surgery proceeded immediately. The operative findings were perforations of the transverse colon and transverse mesocolon. Repair with primary sutures was performed. After surgery, the patient went to the intensive care unit; immediately after her arrival there, intra-abdominal bleeding was confirmed through the drainage bag. Therefore, she underwent a second emergency laparotomy. Vessels near the superior mesenteric artery under the transverse mesocolon were damaged. We could not find the definite bleeding focus because massive bleeding obscured vision, and the patient's blood pressure was unstable. We thus performed resuscitative endovascular balloon occlusion of the aorta (REBOA) intraoperatively. The balloon was placed in zone I of the aorta, but for various reasons, we could not pinpoint the location of the catheter in operating room (Fig. 2.). After bleeder ligation and deflation of the balloon, there was no more active bleeding. The balloon was maintained for 21 minutes

Received: April 5, 2019 Revised: May 8, 2019 Accepted: May 8, 2019

Correspondence to: Pil Young Jung, Department of Surgery, Yonsei university Wonju college of medicine, Wonju Severance Christian Hospital, Trauma center, 220-701, 20 Ilsan-ro, Wonju-si, Gangwon-do, South Korea

Tel: 82-33-741-0882, Fax: 82-33-741-0574, E-mail: surgery4trauma@yonsei.ac.kr

Copyright (C) 2019 Korean Association for Research, Procedures and Education on Trauma. All rights reserved.

@This is an open-access article distributed under the terms of the Creative Commons Attribution Non-Commercial License (http://creativecommons.org/ licenses/by-nc/4.0) which permits unrestricted noncommercial use, distribution, and reproduction in any medium, provided the original work is properly cited 


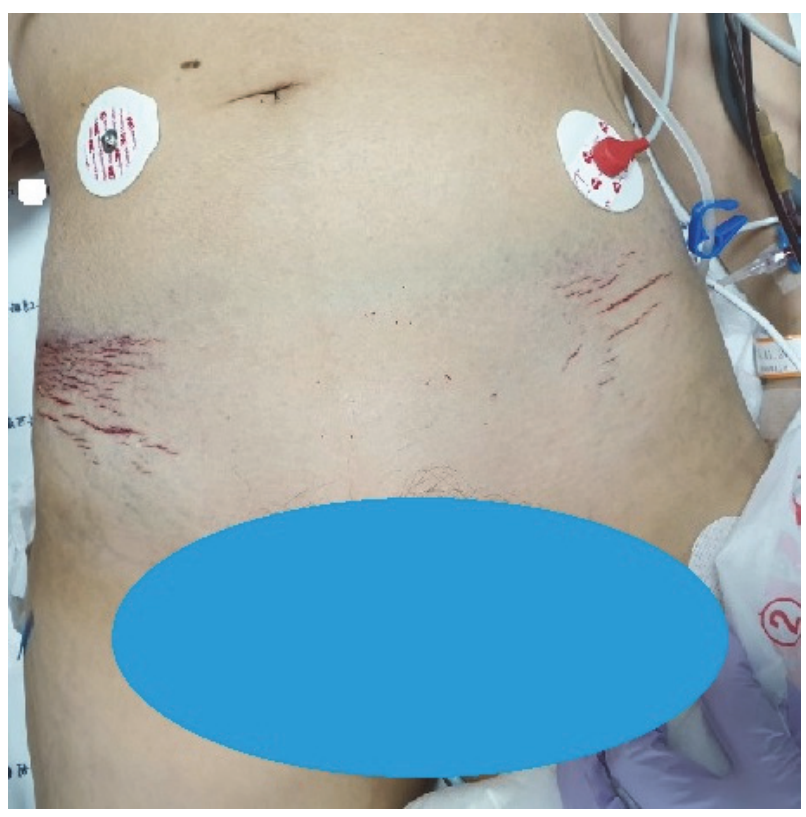

Fig. 1. External photographs, showing the seat belt sign in the lower abdomen.

and 20 seconds and caused total occlusion of aorta. The oozing in the abdominal cavity continued, and so we performed intra-abdominal taping. The next day, the intra-peritoneal packed tapes were removed. Because no more bleeding from the intra-abdominal cavity occurred, the REBOA and sheath were removed. Follow-up abdominal computed tomography (CT) on postoperative days 6,9 , and 20 showed no demonstrable cortical defect in the gastrointestinal tract or extravasation of contrast material. The patient was discharged without any abdomen-related complication on postoperative day 51 .

\section{DISCUSSION}

When the patients are in hemorrhagic shock as a result of massive bleeding, REBOA can be performed in an emergency room or at the patient's bedside. (1) The procedure can produce temporary hemodynamic stability, allowing for definitive injury repair. (5) In this case, blood vessel injury was found during surgery; REBOA enabled the site of injury to be secured. The performance of REBOA during the operation was the reasonable procedure, and it produced a good outcome.

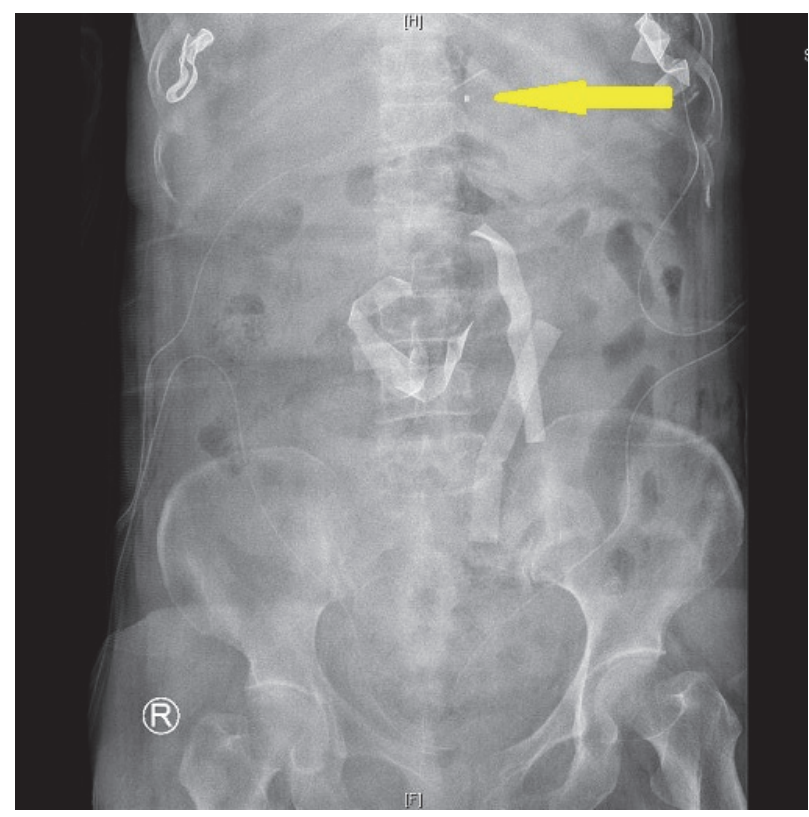

Fig. 2. Postoperative abdominal plain radiograph, showing location of the balloon. (The yellow arrow indicates the tip of the balloon.)

This suggests that REBOA may be appropriate in the treatment of massive bleeding.

\section{Conflict of Interest Statement}

None of authors have a conflict of interest.

\section{REFERENCE}

1. Cannon J, Morrison J, Lauer C, Grabo D, Polk T, Blackbourne L, et al. Resuscitative Endovascular Balloon Occlusion of the Aorta (REBOA) for Hemorrhagic Shock. Mil Med. 2018;183(suppl_2):55-9.

2. Pieper A, Thony F, Brun J, Rodiere M, Boussat B, Arvieux C, et al. Resuscitative endovascular balloon occlusion of the aorta for pelvic blunt trauma and life-threatening hemorrhage: A 20-year experience in a Level I trauma center. J Trauma Acute Care Surg. 2018;84(3):449-53.

3. Sato R, Kuriyama A, Takaesu R, Miyamae N, Iwanaga W, Tokuda $\mathrm{H}$, et al. Resuscitative endovascular balloon occlusion of the aorta performed by emergency physicians for traumatic hemorrhagic shock: a case series from Japanese emergency rooms. Crit Care. 2018;22(1):103.

4. Ordonez CA, Parra MW, Manzano-Nunez R, HerreraEscobar JP, Serna JJ, Rodriguez Ossa P, et al. Intraopera- 
tive combination of resuscitative endovascular balloon occlusion of the aorta and a median sternotomy in hemodynamically unstable patients with penetrating chest trauma: Is this feasible? J Trauma Acute Care Surg. 2018;84(5):752-7.

5. Moore LJ, Brenner M, Kozar RA, Pasley J, Wade CE,
Baraniuk MS, et al. Implementation of resuscitative endovascular balloon occlusion of the aorta as an alternative to resuscitative thoracotomy for noncompressible truncal hemorrhage. J Trauma Acute Care Surg. 2015; 79(4):523-30; discussion 30-2. 